\title{
Radial Diffusion of Negative Ions Produced by Magnetized String-Type $\mathrm{CF}_{4}$ Plasma
}

M. Abid IMTIAZ and Tetsu MIENO*

Department of Physics, Faculty of Science, Shizuoka University, Ooya, Suruga-ku, Shizuoka, 4228529, Japan

Fluorine negative ions $\left(\mathrm{F}^{-}\right)$produced by a column-type magnetized plasma with $\mathrm{CF}_{4}$ gas diffuse radially and accumulate around the plasma column. To explain the features of this accumulation, the radial profiles of the negative-ion density $n_{-}$as functions of discharge parameters are measured using a hot-wire-type Langmuir probe. The modified Bohm theory is applied to evaluate $n_{-}$as $n_{-} / n_{e}>2$ and the negative ions are well confined between two end plates. $n_{-}$around the plasma column decreases with increasing magnetic field from 0.01 to $0.15 \mathrm{~T}$. With increasing pressure from 0.25 to $1.1 \mathrm{~Pa}, n_{-}$around the plasma column gradually decreases.

KEYWORDS: magnetized string plasma, fluorine negative ion, Langmuir probe, radial diffusion, carbon tetra fluoride, ion source

\footnotetext{
*To whom correspondence should be addressed. E-mail: piero@sannet.ne.jp
} 
In conventional plasma etching methods, charging in microscopic etching holes would break insulation layers. ${ }^{1)}$ Notching (local sidewall etching) due to the charging effect obstructs precise silicon etching. ${ }^{2,3)}$ To avoid these problems, silicon etching using negative ions has been investigated. ${ }^{4,5)}$ A negative-ion beam with a considerably high ion flux applicable to silicon etching is a strong candidate for the superior production of ultralarge-scale integrated circuits (ULSIs) to avoid charge accumulation in 10-nm-scale etching holes. For this purpose, a column-plasma-type negative-ion source has been proposed and developed, ${ }^{6,7)}$ by which halogen negative ions can be produced and alkali-metal impurities can be excluded. In these sources, negative ions are more likely to accumulate around the plasma column ${ }^{8)}$ because of sufficient confinement along a magnetic field. In a hollow-type electron cyclotron resonance (ECR) plasma, high-density negative ions have accumulated in the central region along a magnetic field. By utilizing a multistring-type plasma, a large volume negative-ion source has been developed. ${ }^{9)}$

Here, to obtain the optimum conditions for accumulating negative ions, the diffusion features of negative ions and electrons across the magnetic field have been investigated experimentally. Fluorine negative ions $\left(\mathrm{F}^{-}\right)$have been produced using a magnetized $\mathrm{CF}_{4}$ plasma by injecting a string-type electron beam along a magnetic field, and their properties as functions of magnetic field, gas pressure and beam current have been investigated.

A simple density model of $\mathrm{CF}_{4}$ plasma is considered, where there are three dominant charged species: $\mathrm{CF}_{3}^{+}$(created by electron impact ionization, e $+\mathrm{CF}_{4} \rightarrow \mathrm{CF}_{3}^{+}+\mathrm{F}+2 \mathrm{e}$ ), $\mathrm{F}^{-}$(created by dissociative attachment, e $+\mathrm{CF}_{4} \rightarrow \mathrm{CF}_{3}+\mathrm{F}^{-}$), and electrons. Thermal electrons in the plasma are produced by injecting an electron beam along the magnetic field with $100 \mathrm{eV}$ of energy, part of which is lost at the end electrodes after diffusing along the magnetic field, and attach to $\mathrm{CF}_{4}$ molecules. On the other hand, the produced negative ions $\left(\mathrm{F}^{-}\right)$are confined well along the magnetic field by sheath potentials at the end plates, and are partially lost by recombining with positive ions. Around the column-type plasma, the radial diffusion speeds of electrons and negative ions 
are very different, which causes the production of ion-ion plasmas. The perpendicular diffusion coefficient of an electron is much smaller $(\sim 1 / 10$ times $)$ than that of a negative ion.

In this model, electrons are produced only in the electron beam region, and therefore become extinct at the end plates with an absorption probability or diffuse across the magnetic field. In a steady-state plasma, the continuity equation for electrons can be written as

$$
K_{i z} n_{E B} n_{0}+D_{\perp e} \nabla^{2} n_{e}-\alpha n_{e}=0
$$

where $K_{i z}$ is the ionization reaction rate constant, $n_{E B}$ is the beam electron density, $n_{0}$ is the neutral molecule density, $D_{\perp e}$ is the radial diffusion coefficient for an electron, $\alpha$ is the electron absorption coefficient, which shows sum of the time-averaged absorption probability at the end plates and the probability by the ion-ion attachment, and $n_{e}$ is the thermal electron density. From ref. $10, K_{i z}=$ $2.98 \times 10^{-13} \mathrm{~m}^{3} / \mathrm{s}$. Equation (1) is solved in a cylindrical coordinate system using Mathematica software (Wolfram Research Inc., ver. 5.2), and thus a radial profile of the electron density is obtained.

Negative ions, in this model, are assumed to be produced through the dissociative attachment, bounce at the end plates, diffuse across the magnetic field and therefore become extinct partly by recombination with positive ions. The continuity equation for negative ions in a steady-state plasma can be written as

$$
K_{a t t} n_{e} n_{0}+D_{\perp-} \nabla^{2} n_{-}-K_{r e c} n_{-} n_{+}=0
$$

where $K_{a t t}$ is the dissociative attachment coefficient of $\mathrm{F}^{-}$ions production, $K_{r e c}$ is the ion-ion recombination coefficient , $D_{\perp_{-}}$is the radial diffusion coefficient of negative ions, and $n_{+}$is the positive-ion density. From ref. 11 and $12, K_{\text {att }}=7.57 \times 10^{-17} \mathrm{~m}^{3} / \mathrm{s}$ and $K_{r e c}=5.0 \times 10^{-13} \mathrm{~m}^{3} / \mathrm{s}$. This equation is also solved using Mathematica software and the radial profile of the negative-ion density is obtained.

In the Langmuir probe measurement, the presheath potential $\Delta \emptyset_{p s}$ in front of an electrode is reduced owing to negative ions, and a fraction of the negative ions is reflected in the presheath re- 
gion. This condition is referred to as "the modified Bohm condition". 13) The effect of negative ions on the generation of a presheath $\left(n_{-} / n_{e} \geq 2\right)$ and $n_{e b}+n_{-b}=n_{+b}\left(n_{e b}\right.$ : electron density in the bulk plasma, $n_{-b}, n_{+b}$ : negative-ion density and positive-ion density in the bulk plasma) is considered. At the reference position, the charge neutrality condition $n_{e 0}=n_{+0}$ is assumed, where $n_{e 0}$, $n_{+0}$ are the electron density and positive-ion density, respectively, and the negative-ion density is much smaller. The density ratio of negative-ion density to electron density in the bulk plasma is written as ${ }^{7,13)}$

$$
\frac{n_{-b}}{n_{e b}} \approx \frac{J_{+s} / n_{e b}}{J_{+s 0} / n_{e 0}} \exp \left(\frac{e \gamma \Delta \emptyset_{p s}}{\kappa T_{e b}}-\frac{1}{2}\right) \sqrt{\frac{\kappa T_{e 0}}{2 e \Delta \emptyset_{p s}}}-\exp \left\{\frac{e \Delta \emptyset_{p s}(\gamma-1)}{\kappa T_{e b}}\right\}
$$

where $\gamma=T_{e b} / T_{-b}$ (ratio of electron temperature to negative-ion temperature in the bulk plasma). $J_{+s 0} / n_{e 0}$ (ratio of positive-ion saturation current of the probe to electron density) and $T_{e 0}$ (electron temperature) are measured at a reference position in the radial center, where $n_{-b} / n_{e b} \ll 1 . \quad n_{-b} / n_{e b}$ is obtained using eq. 6 and Fig. 3 of ref. 7, where the asymptotic method using the graph is utilized. In this experiment, the effect of positive-ion temperature can be neglected in the evaluation of $n_{-b} / n_{e b}$ because the ion flow to the probe with $\kappa T_{-b} \sim 0.1 \mathrm{eV}^{14,15)}$ has little influence on sheath generation.

Figure 1 shows a schematic diagram of the experimental setup. A metal chamber of $2.0 \mathrm{~m}$ length and $21 \mathrm{~cm}$ diameter is set across eight solenoid coils producing a magnetic field of $0-0.15$ T. An electron beam of $10 \mathrm{~mm}$ diameter is injected by an electron beam source (placed at the axial center about $85 \mathrm{~cm}$ from the chamber end), which has a beam energy of $\Psi_{B E}=100 \mathrm{eV}$ and a current of $I_{B E}=0-0.05 \mathrm{~A}$. The anode of the beam source $(20 \mathrm{~cm}$ in diameter $)$ with a gridded hole of 10 $\mathrm{mm}$ diameter is grounded. At about $77 \mathrm{~cm}$ from the beam source, an electrically grounded end plate, $20 \mathrm{~cm}$ in diameter, is placed. Hot-wire-type Langmuir probes, made of Ta wire of $0.2 \mathrm{~mm}$ diameter, shaped as half-circles of $1.5 \mathrm{~mm}$ diameter are used for the diagnosis of the plasma. Here, results are obtained using the probe placed $21 \mathrm{~cm}$ from the anode plate. 
The radial density profiles of electrons, $n_{e}$, and negative ions, $n_{-}$, obtained experimentally and using the model calculations with eqs. (1) and (2) are shown in Fig. 2. In this case, $B=0.03 \mathrm{~T}$, $p=0.6 \mathrm{~Pa}, \Psi_{B E}=100 \mathrm{eV}$ and $I_{B E}=0.05 \mathrm{~A} . \quad$ In the calculation of eq. (1), $D_{\perp e}=0.040 \mathrm{~m}^{2} / \mathrm{s}$ (ref. 16) and $\alpha=2.0 \times 10^{3} \mathrm{~m}^{2} / \mathrm{s}$ are utilized, and adequate boundary conditions are used. The first term of eq. (1) can be omitted as it is assumed that no electrons are produced outside the beam radius $(r=5$ $\mathrm{mm}$ ). In the calculation of eq. (2), $D_{\perp-}=0.50 \mathrm{~m}^{2} / \mathrm{s}$ (ref. 16), $K_{r e c}=5.0 \times 10^{-13} \mathrm{~m}^{3} / \mathrm{s}$ (ref. 12) and $n_{-} \approx n_{+}$are utilized. As zero production of negative ions outside the beam diameter is assumed, the first term of eq. (2) can be omitted.

It can be confirmed that the electrons become extinct significantly outside the beam diameter, whereas the negative-ion density radially decreases gradually due to radial diffusion and axial confinement at the end plates. Therefore, around the string plasma, ion-ion plasmas with high $n_{-}$have been generated. However, there is a discrepancy between the experiment and the calculation of the radial profiles of $n_{-}$. It is conjectured that the potential structure or some instabilities suppress the radial diffusion.

The radial profiles of the plasma potential $\emptyset_{p}$ and the floating potential $\emptyset_{f}$ at $p=0.6 \mathrm{~Pa}$ and $B=0.03 \mathrm{~T}$ are shown in Fig. 3. The plasma potential throughout the radial distribution is positive, which confines the negative ions at the end plates.

The magnetic field dependences of $n_{-}$and $n_{e}$ at $r=0,+5$, and $-5 \mathrm{~cm}$ are shown in Fig. 4, where $p\left(\mathrm{CF}_{4}\right)=0.6 \mathrm{~Pa}, \Psi_{B E}=100 \mathrm{eV}$ and $I_{B E}=0.05 \mathrm{~A}$. It is found that $n_{-}$decreases with an increase in $B$ at $r=+5$ and $-5 \mathrm{~cm}$. By increasing the magnetic field, $n_{e}$ at $r=0$ increases gradually, whereas at $r=+5$ and $-5 \mathrm{~cm}, n_{e}$ remains almost constant.

The pressure dependences of $n_{-}$and $n_{e}$ at $r=0,+5$, and $-5 \mathrm{~cm}$ are shown in Fig. $5 . n_{-}$ gradually decreases at $r=+5$ and $-5 \mathrm{~cm}$, with an increase in $p\left(\mathrm{CF}_{4}\right)$, where $B=0.03 \mathrm{~T}, \Psi_{B E}=100$ 
$\mathrm{eV}$ and $I_{B E}=0.05$ A. $n_{e}$ at $r=0$ slowly decreases with increasing $p\left(\mathrm{CF}_{4}\right) . n_{e}$ at $r=+5$ and $-5 \mathrm{~cm}$ remains almost constant with increasing $p$.

Fluorine negative ions, $\mathrm{F}^{-}$, produced by the string-type magnetized plasma with $\mathrm{CF}_{4}$ gas diffuse radially and are confined in the magnetic field. The radial diffusion of electrons is consistent with the model calculation, whereas the radial diffusion of $\mathrm{F}^{-}$is not well clarified by the model calculation, for which a study is underway. The electron beam current, magnetic field and gas pressure are the controlling parameters for negative-ion production. In this experiment, by decreasing $B$ and decreasing $p$, a higher accumulation of $\mathrm{F}^{-}$ions is obtained.

In order to obtain a large-diameter negative-ion source, a multistring-type plasma can be used $^{9)}$, where negative ions accumulate among the string-type-plasma columns. By using a specially designed grid with an acceleration electric potential and a converging magnetic field, only negative ions can be extracted for a large-diameter negative-ion beam, which will be reported in the future after further study.

This work is partly supported by a Grant-in-Aid from the Ministry of Education, Culture, Sports, Science and Technology, Japan. 
1) K. P. Cheung and C. P. Chang: J. Appl. Phys. 75 (1994) 4415.

2) H. Ootera, T. Oomori, M. Tuda, and K. Namba: Jpn. J. Appl. Phys. 33 (1994) 4276.

3) T. Kinoshita, M. Hane, and J. P. McVittie: J. Vac. Sci. \& Technol. B 14 (1996) 560.

4) H. Shindo, Y. Sawa, and Y. Horiike: Jpn. J. Appl. Phys. 34 (1995) L925.

5) T. Shibayama, H. Shindo, and Y. Horiike: Plasma Sources Sci. Technol. 5 (1996) 254.

6) R. Kawai and T. Mieno: Jpn. J. Appl. Phys. 36 (1997) L1123.

7) T. Mieno and A. Ogawa: Jpn. J. Appl. Phys. 38 (1999) 4586.

8) N. Sato: Plasma Sources Sci. Technol. 3 (1994) 395.

9) M Abid Imtiaz, S. Tsuruta, and T. Mieno: submitted to Plasma Sources Sci. Technol.

10) L. G. Christophorou and J. K. Olthoff: Fundamental Electron Interactions with Plasma Processing Gases (Kluwer Academic/ Plenum Publications, New York, 2004) p. 176.

11) L. G. Christophorou and J. K. Olthoff: Fundamental Electron Interactions with Plasma Processing Gases (Kluwer Academic/ Plenum Publications, New York, 2004) p. 199.

12) A. Kono, M. Haverlag, G. M. W. Kroesen, and F. J. de Hoog: J. Appl. Phys. 70 (1991) 2939.

13) M. A. Lieberman and A. J. Lichtenberg: Principles of Plasma Discharges and Materials Processing (John Wiley \& Sons, New York, 1994) p. 167.

14) T. Nakano, H. Ohtake and S. Samukawa: Abstr. 189th Meet. Electrochemical Society, Los Angeles, 1996, Vol. 96-1, p. 228.

15) H.- J. Woo, K.- S. Chung and T. Lho: Proc. 6th Int. Conf. Reactive Plasmas, Sendai, 2006, p. 779.

16) F. F. Chen: Introduction to Plasma Physics and Controlled Fusion (Plenum Press, New York, 1984) 2nd ed., p. 172. 


\section{Figure captions}

Fig. 1. Schematic diagram of DC plasma experiment.

Fig. 2. Radial profiles of $n_{e}$ (a) and $n_{-}$(b) obtained by experiments and using model calculations. $\quad B=0.03 \mathrm{~T}$ and $p=0.6 \mathrm{~Pa}$, measured at $z=21 \mathrm{~cm}$ from the anode.

Fig. 3. Radial distributions of $\varnothing_{p}$ and $\varnothing_{f} . \quad B=0.03 \mathrm{~T}$ and $p=0.6 \mathrm{~Pa}$.

Fig. 4. B dependences of $n_{-}$(a) and $n_{e}$ (b) at $r=0,+5$, and $-5 \mathrm{~cm} . \quad p=0.6 \mathrm{~Pa} . \quad z=21$ $\mathrm{cm}$ from the anode.

Fig. 5. $p$ dependences of $n_{-}(\mathrm{a})$ and $n_{e}(\mathrm{~b})$ at $r=0,+5$, and $-5 \mathrm{~cm} . \quad B=0.03 \mathrm{~T} . \quad z=21$ $\mathrm{cm}$ from the anode. 


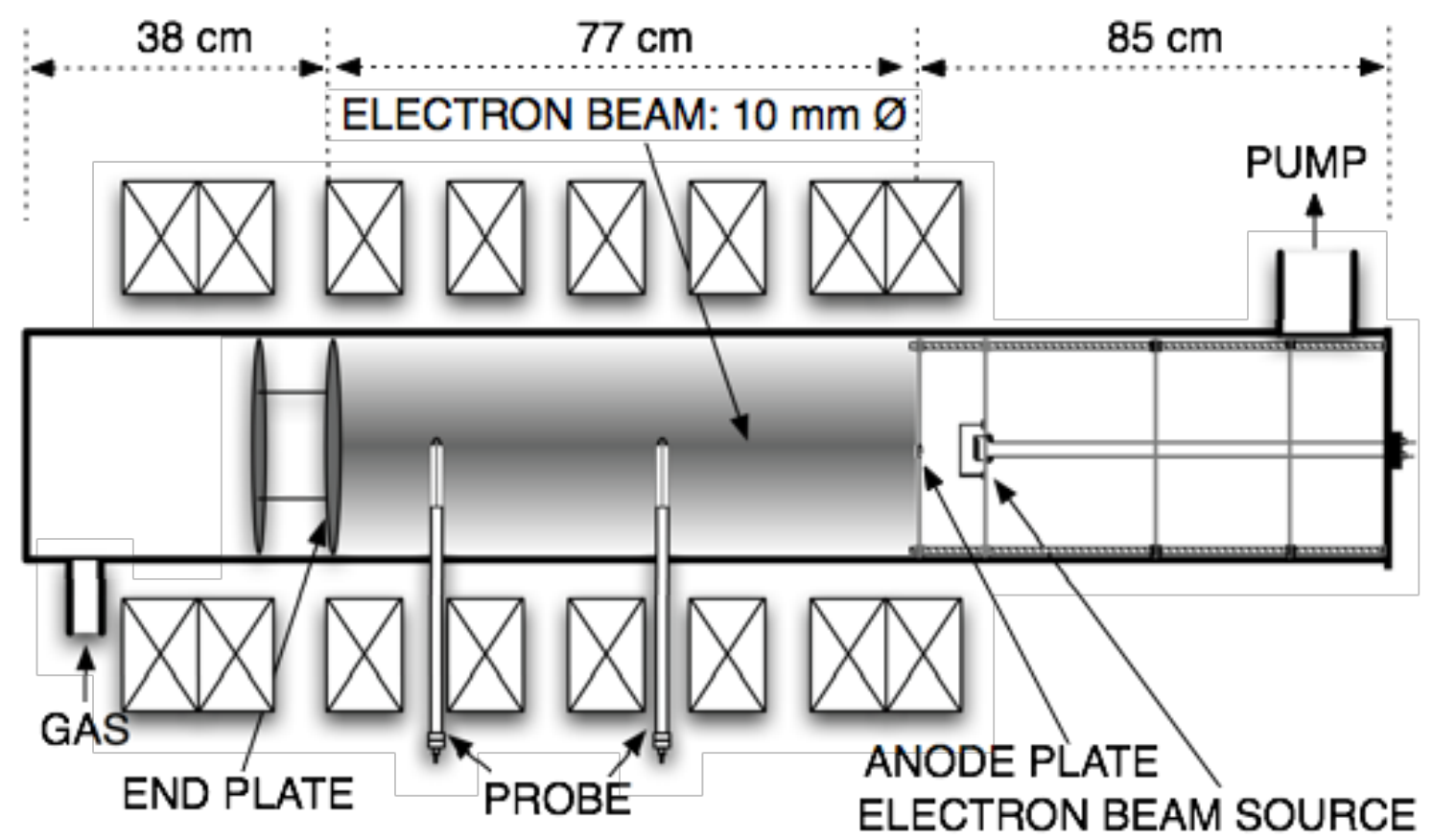

Fig. 1. Schematic diagram of DC plasma experiment. 


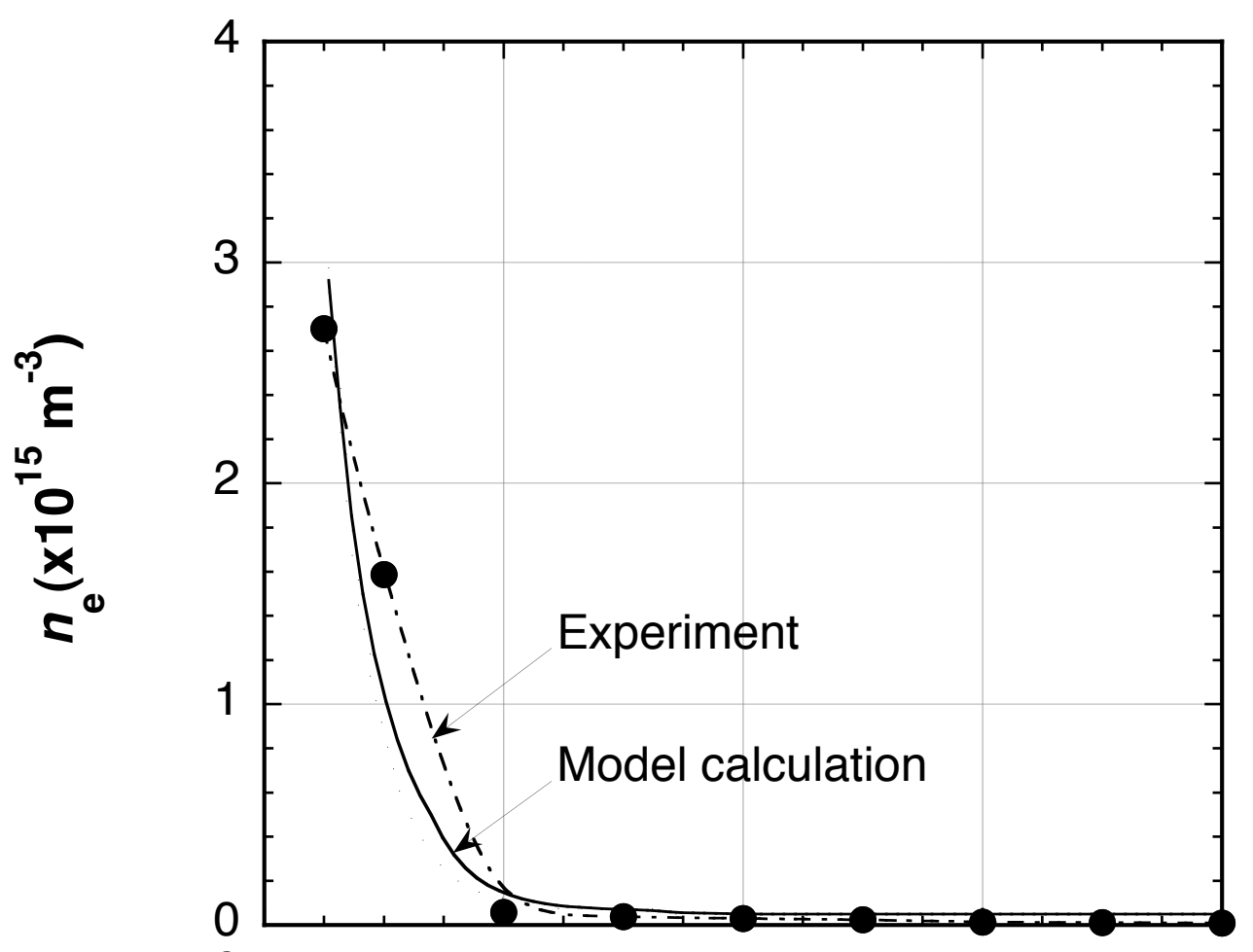

(a)

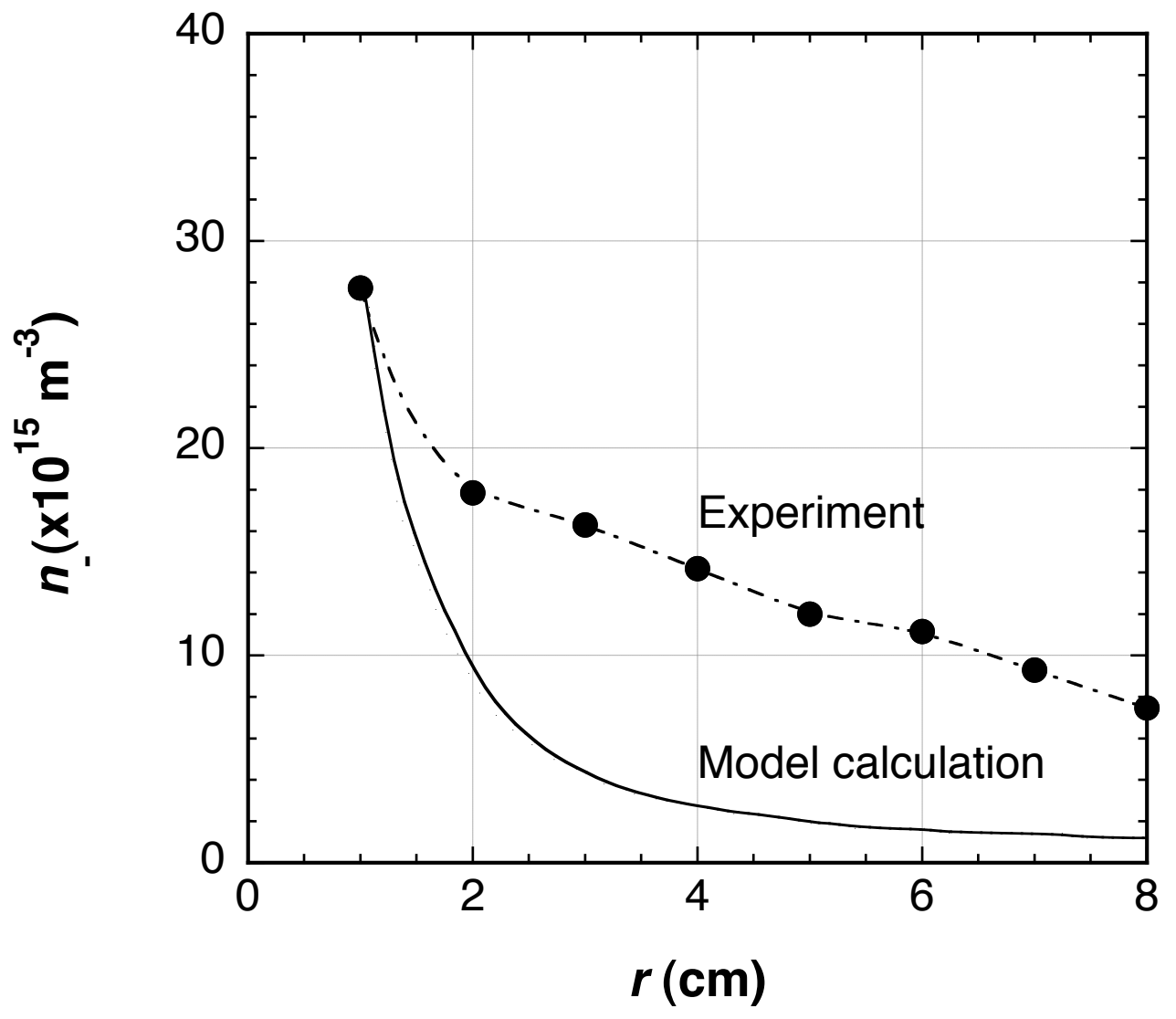

(b)

Fig. 2. Radial profiles of $n_{e}$ (a) and $n_{-}$(b) obtained by experiments and using model calculations. $B=0.03 \mathrm{~T}$ and $p=0.6 \mathrm{~Pa}$, measured at $z=21 \mathrm{~cm}$ from the anode. 


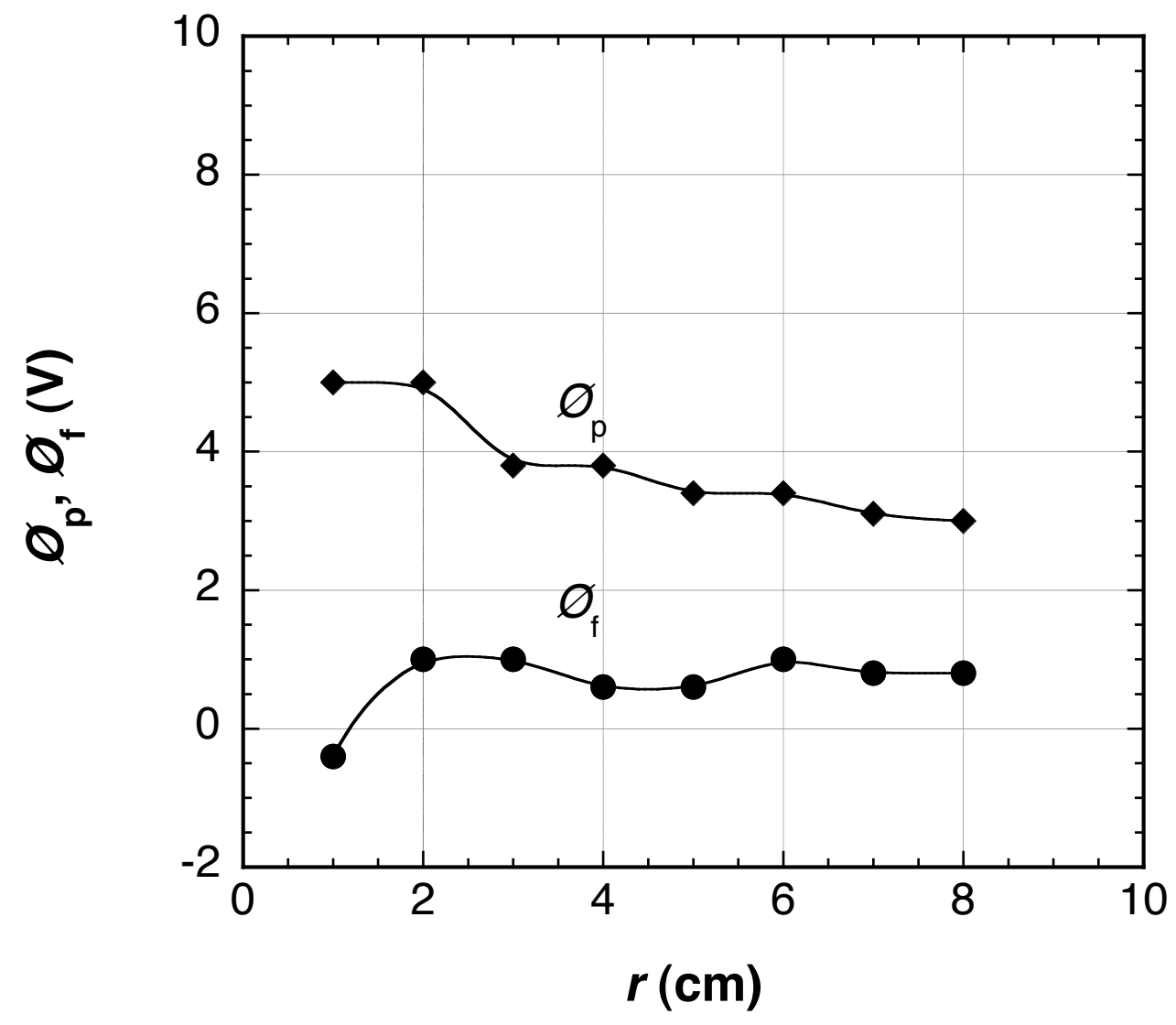

Fig. 3. Radial distributions of $\varnothing_{p}$ and $\varnothing_{f} . \quad B=0.03 \mathrm{~T}$ and $p=0.6 \mathrm{~Pa}$. 


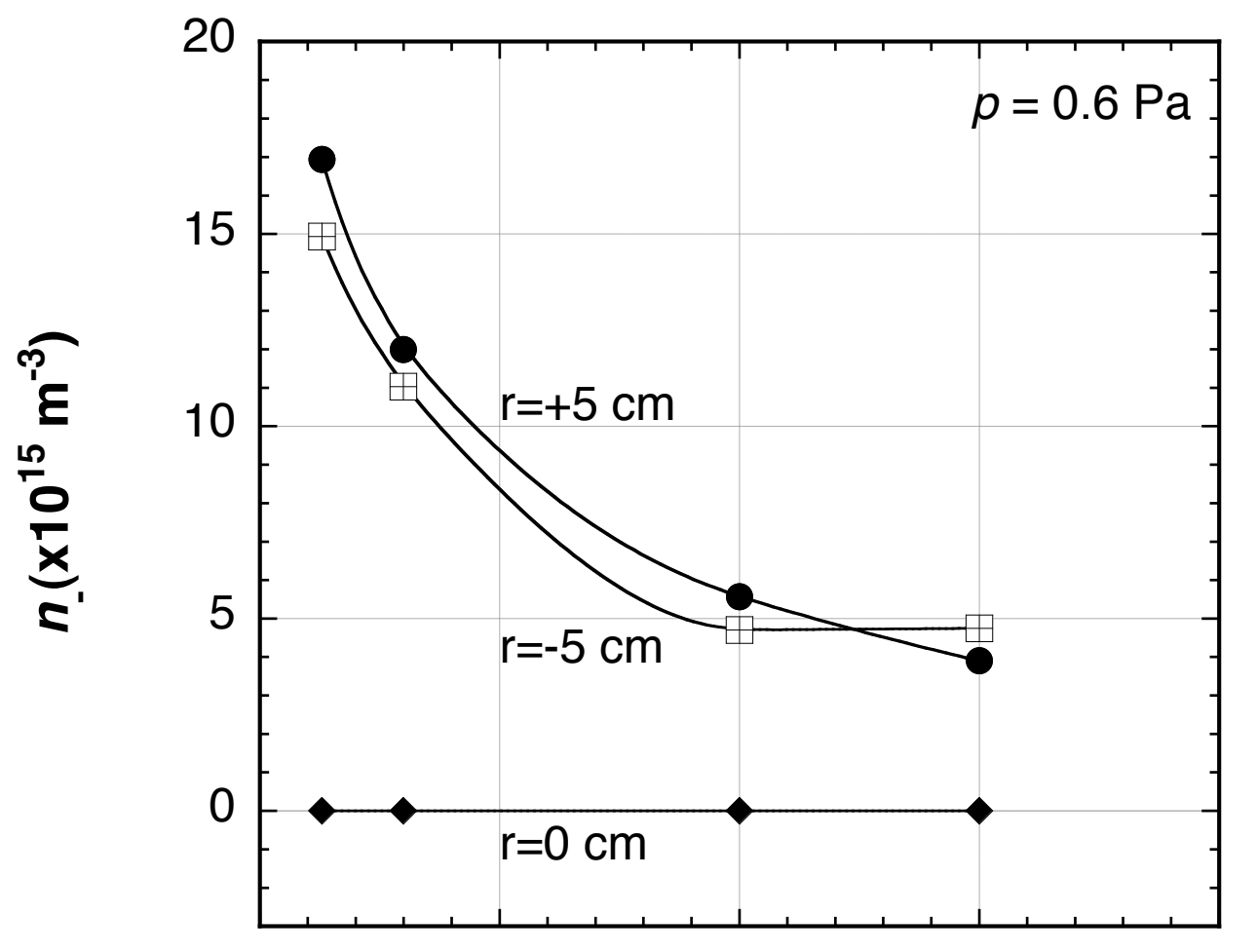

(a)

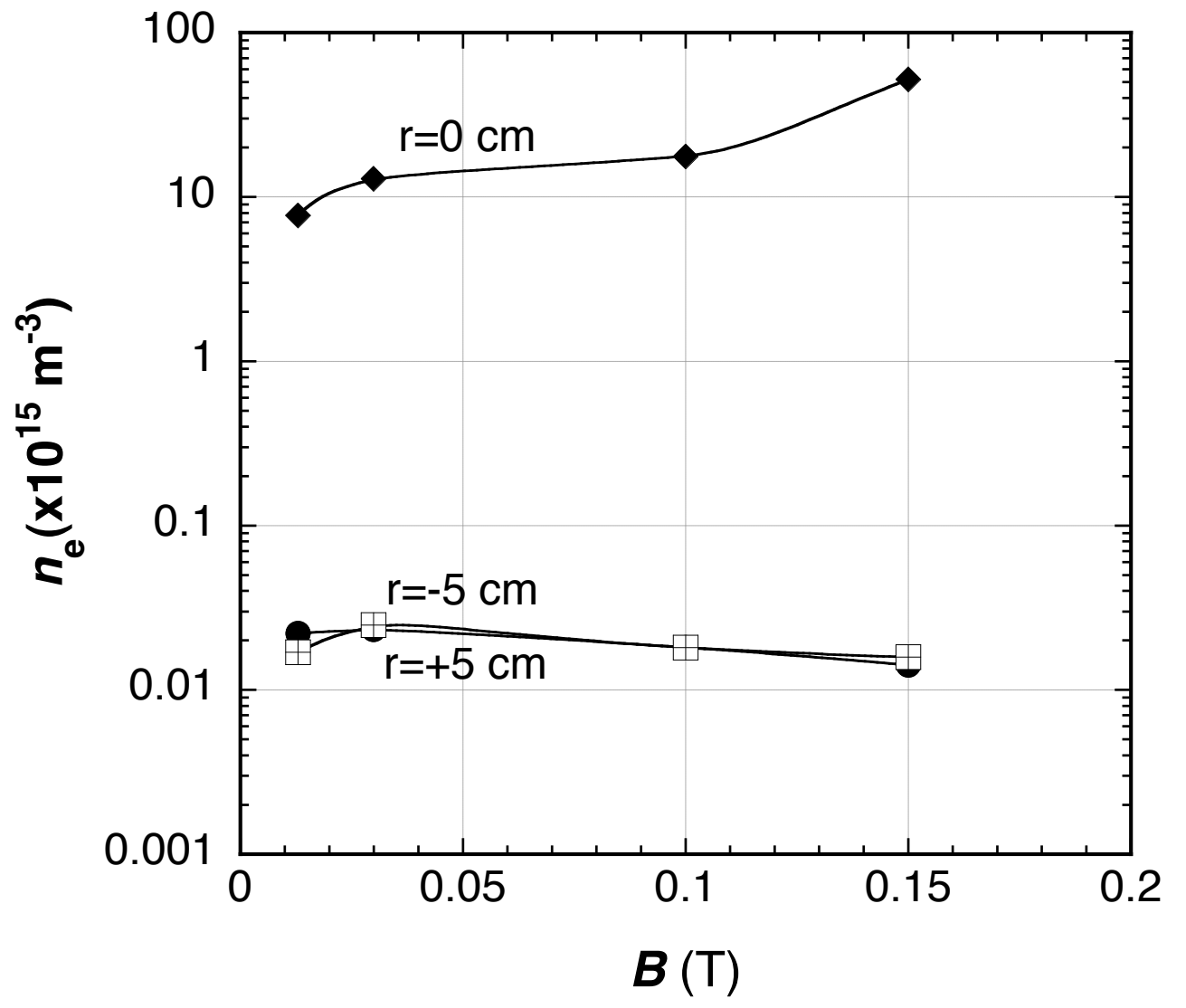

(b)

Fig. 4. $\quad B$ dependences of $n_{-}$(a) and $n_{e}$ (b) at $r=0,+5$, and $-5 \mathrm{~cm} . \quad p=0.6 \mathrm{~Pa} . \quad z=21$ $\mathrm{cm}$ from the anode. 


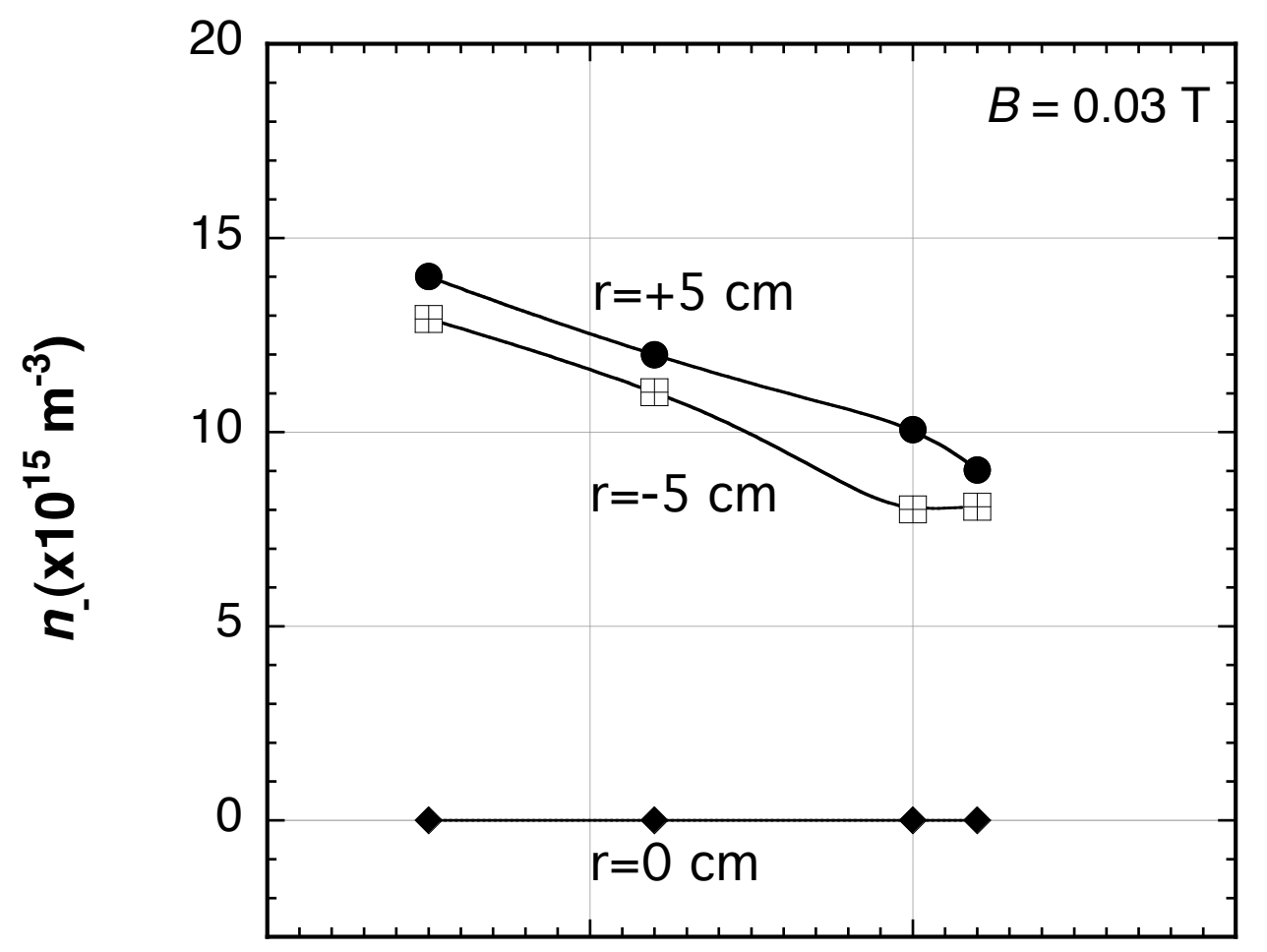

(a)

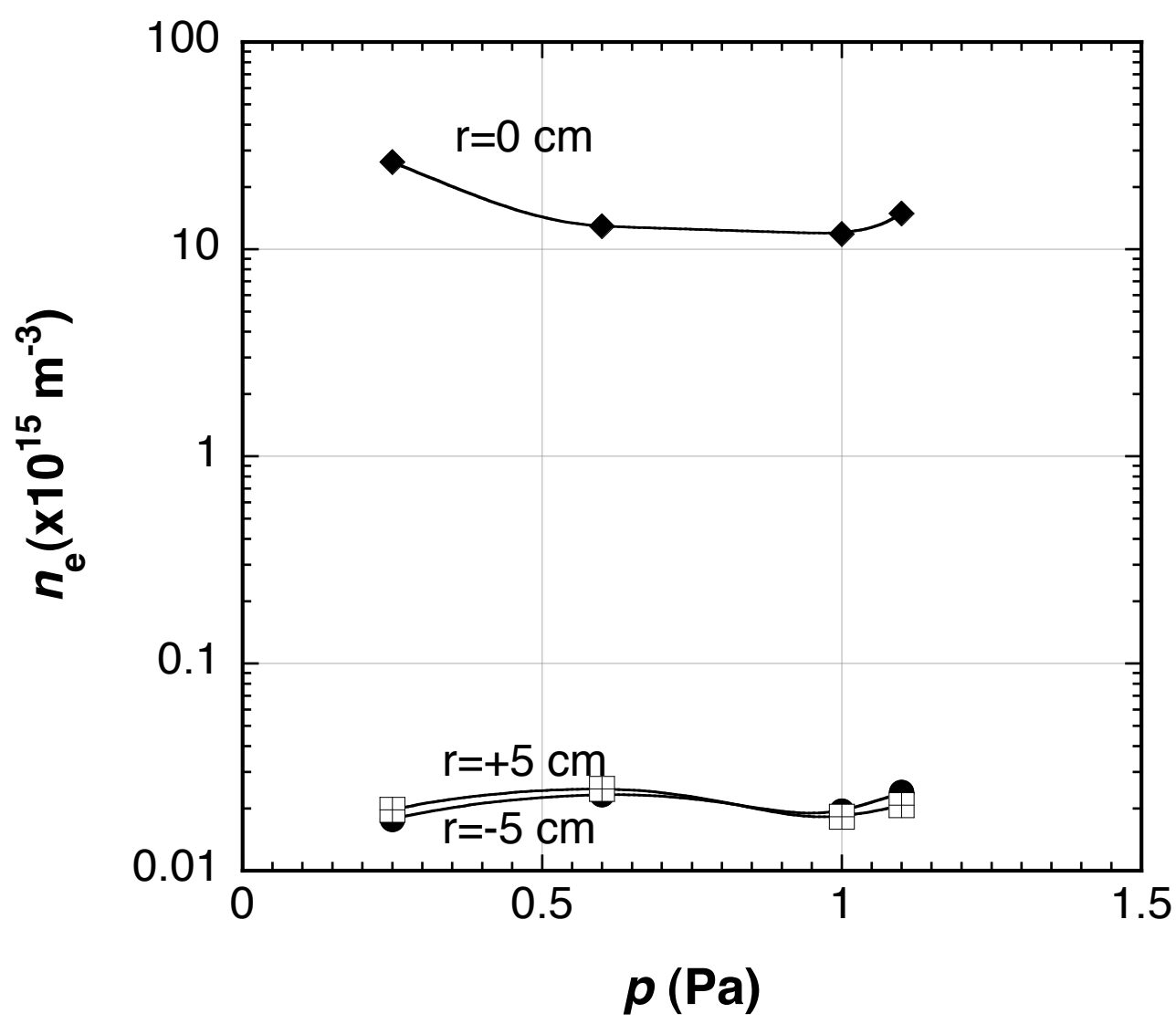

(b)

Fig. 5. $\quad p$ dependences of $n_{-}$(a) and $n_{e}$ (b) at $r=0,+5$, and $-5 \mathrm{~cm} . \quad B=0.03 \mathrm{~T} . \quad z=21$ $\mathrm{cm}$ from the anode. 